\title{
Téoros
}

Revue de recherche en tourisme

\section{Prospective touristique locale et régionale, 1995-2010 : scénarios tendanciels et exploratoires}

\section{Jean-Claude Jay-Rayon et Brigitte Morneau}

Volume 12, numéro 2, juillet 1993

La prévision-prospective du tourisme

URI : https://id.erudit.org/iderudit/1078026ar

DOI : https://doi.org/10.7202/1078026ar

Aller au sommaire du numéro

Éditeur(s)

Université du Québec à Montréal

ISSN

0712-8657 (imprimé)

1923-2705 (numérique)

Découvrir la revue

Citer cet article

Jay-Rayon, J.-C. \& Morneau, B. (1993). Prospective touristique locale et régionale, 1995-2010 : scénarios tendanciels et exploratoires. Téoros, 12(2), 44-51. https://doi.org/10.7202/1078026ar d'utilisation que vous pouvez consulter en ligne. 


\section{Prospective touristique locale et régionale, 1995-2010 : scénarios tendanciels et exploratoires \\ Jean-Claude Jay-Rayon et Brigitte Morneau*}

Commele précisait avec justesse Roger Sauvé, *futurist manager», lors du Rural Exchange 1993 , tenu à Hull, «... nous ne sommespasintellectuellementpoussésvers le futur mais, tout simplement et physiquement aspirés par lui». C'est sans doute pour cette raison que le *Future Pull» est angoissant pour nombre d'entre nous, et qu'en tourisme, on préfere offrir aux visiteurs une très grande quantité de produits *passéistes* plutôt que *futuristes* $*$ (1).

Cela comprend la plupart des musées, sauf les contemporains, quelques zoos, le patrimoine architectural et les centres d'interprétation historiques, les milieuxnaturels... intouchables, la reconstitution de déoors $\mathrm{du}$ \&bon vieux temps ${ }^{(2)}$, les modes de vie ancestraux des Amérindiens, etc. En bref, le tourisme s'abreuve surtout au mythe du «paradis perdu*, mais néglige souvent son futur, à l'exception peut-ểtre d'sEpcot Centers aux Etats-Unis, qui a décidé et réussi à projeter des millions d'individus au sein du prochain millénaire tout en leur rappelant les origines.

Devant cet état de fait déjà souligné par le géographe Luc Bureau de l'Université Laval, tenter de dégager une prospective touristique cohérente concernant les régions du Québec au sein des horizons de 2010, exige à la fois une prudence et un décodage précis des tendances déjà à l'oeuvre au niveau international et mondial. Car, ces trois pbénomènes: la mondialisation, linternationalisation des marchés et la relative standardisation des produits concernent deja directement la moindre localité qui veut diversifier son économie par le tourisme.

\footnotetext{
Monsieur Jean-Claude Jay-flayon est chercheurconcepteur et maitre de conférence. Madame Brigitte Morneau est associte experte au sein de la firme Tangram et se spécialise dans la mise en valeur touristique d'environnements naturels et culturels.
}

Quant à la prospective elle-même, sujet quel'on aborde de plus en plus sous l'aspect de la planification stratégique à moyen terme, il faut en cette matière éviter d'agir en pur visionnaire en prévoyant l'avenir par intuition et fabrication artificielle de scénarios, ou encore à l'inverse, projeter de façon linéaire le présent dans le futur. Par contre, on peut avoir connaissance de l'émergence du futur au sein de plusieurs recherches et en maints endroits et de ce fait, en analyser les conséquences probables. Telle est notre approche, ce qui nous l'espérons, noussituera à l'abride la «pensée magique», si courante de nos jours.

\section{Un état de situation... qu'il fasse beau ou qu'il pleuvel}

En 1990, la masse touristique québécoise, tous marchés confondus, s'élevait à 19577 000 personnes-jours et rapportait comme recettes, environ 4033 milliards de dollars. Plus précisément, les Québécois représentaient, au niveau interrégional, $67,4 \%$ de tous les touristesduQuébec, les Américains, $14,6 \%$, les autres Canadiens, $14,1 \%$ et les résidents des autres pays étrangers que les États-Unis, 3,9\%.

Sur le plan des recettes touristiques, les Québécois ne rapportaient pourtant que $34,4 \%$ des recettes totales, les Américains $16,6 \%$, les autres Canadiens, $11,5 \%$, les résidents des pays autres que les ÊtatsUnis, $10 \%$, etc. Une certaine lecture de ces données permet alors de constater que le volume touristique étranger, s'élevant seulement à $32,5 \%$ du volume global rapporte quand même $38,1 \%$ des recettes. A l'inverse, le tourisme interrégional (intérieur) qui représente $67,4 \%$ de tous les touristes au Québec, ne rapporte que 34,4 $\%$ des recettes totales.

Aux dires mềme du ministère du Tourisme du Québec, l'industrie touristique affiche une performance globale relativement stable depuis la dernière décennie.Certains la qualifient de stagnante! On y observe, à la fois, une baisse des nuitéesetune tendance généraleà écourter la durée des séjours tant au Canada qu'au Québec. Et comme le mentionne Louis Jolin de l'UQAM, les dientèlesextérieures les plus traditionnelles du Québec diminuent: la clientèle américaine de $2,4 \%$ en 1992 par rapportà 1991 ; et il y a eu une diminution de $12,9 \%$ des Ontariens au Québec entre 1988 et 1990. Toutefois, on constate une augmentation de $18,8 \%$ des visiteurs français en 1991 ; une clientèle qui, dans la masse des recettes touristiques ne dépasse pas les $3 \%$.

À l'inverse, depuis 1980, les arrivées touristiquesà l'échellemondialeont augmenté de $65 \%$ et les recettes provenant des voyages internationaux ont fait un bond de $174 \%$. Par contre, au même moment, le Canada et le Québec perdaient du terrain sur le marché mondial.

Selon le *Conference Board of Canada», le déficit touristique augmentera de plus de 500 millions dedollarsen 1993 pours'établir à 9,5 milliards en 1994. Au Québec, l'analyste économiste du journal $\mathbf{L a}$ Presse, Claude Picher, évaluaità 2 milliards dedollars le déficitdela balance budgétaire. Ce qui, d'après lui, créerait 60000 emplois à l'extérieur de la province, aux États-Unis, en Ontario...

Devant ces faits, il est à penser que les régions et les localités cherchant une diversification économique rapideouétant en voie de restructuration, auraient avantage à bien évaluer la réalité touristique dans son ensemble au moment même où elles vont décider de leurs axes de développement et de leurs plans stratégiques pour les prochaines années. L'idéal serait à court terme de redresser le produit touristique de façon énergique afin d'endiguer de manière significative le flot de Canadiens et de Québécois fausant des voyager au Sud(3). Ou encore, que notre part du marché international augmente sensiblement.

Là se situe un questionnement qui entraînerait deux types d'actions et concernerait directement le niveau régional et local. Devra-t-on continuer à offrir tous les produits régionaux existants sans discernement par une mise en marché encore 
plus agressive? Ou devra-t-on d'abord, dans de nombreux cas, renouveler le produit touristique local de façon à l'épurer et à le rehausser pour qu'il puisse devenir plus performant et concurrentiel au niveau intersectoriel, interrégional, intercanadien et international? ${ }^{(4)}$

De l'avis de plusieurs, une grande partie de la problématique actuelle reposerait sur une faiblesse certaine du produit touristique régional et local, laquelle serait involontairement augmentée par une mise en marché très performante. En d'autres mots, le produit touristique canadien et québécoisne posséderait pas assez de valeur ajoutée $e^{(5)}$ et il apparaîtrait dans plusieurs cas sous l'aspect de biens et services en déclin. Ceci est malheureux car, il faut en convenir, dans ce domaine, ce n'est ni $l e$ potentid culturel ni naturel qui manque au Québec mats, une tranformation et une nouvelle mise en valeur concurrentielle de ceux-ci...

En conséquence, et mềme si le travail à effectuer pourra paraître arduà plusieurs, il faudra au cours des années à venir éviter de sombrer dans une vision pessimiste basée sur la situation présente et passée. Le tourisme au Québec est bien vivant, il occupe la sixième place parmi nos exportations et crée environ 56700 emplois directs et 17000 indirects.

\section{L'environnement... matière première touristique de l'avenir}

En 1979, René Dubos dans son livre intitulé Choisir d'être humain disait en parlant d'un certain *Rousseauismes, *L'homme dela caverne està la mode mais pour de mauvaises raisons». Il sous-entendait par là que le retour aux sources, l'homme naturel nouveau, ou l'explication quel'on pouvait donner d'un simple weekend bucolique en campagne dans les années 1970 , n'avait rien à voir avec le bio-comportementdel'individu, sa véritablenature et ses environnements privilégiés. En fait, il s'agissait d'une *natures inventée de toutes pièces, sans assises scientifiques et sans grande symbolique.

Il en est de même de la notion d'environnement utilisée aujourd'hui, et qui est si large et globale que l'on s'y perd en... planétarisme. Au sein de cet enchevêtrement systêmique, où tout est dans tout, le touriste comme individu, groupe ou agrégat $t^{(6)}$ (petite mase) préférera de plus en plus une approche pragmatique et concrète qui Leconcernera sensoriellement et de facon directe. Ce qu'il souhaitera, c'est un environnementcaractérisé à la fois culturel etnaturel, à parcourir brièvement, et à vivre temporairement. Le touriste veut vire l'emvironinement at cette tendance ne fera que croitre au cours de la prochaine décennie?

En ce sens, tout le monde semble déjà acoorder la plus haute importance à la qualité esthétique(s) du milieu de vie temporaire du touriste et ce, jusqu'aux années 2020 environ, si l'on considère le temps qu'il faudra mettre pour corriger les graves blessures environnementales du $\mathrm{XX}^{\mathrm{e}}$ siècle présentes dans presque toutes les régions. Cette question est si primordiale qu'au cours des tendances pour 1993, exposées par le *Conference Board of Canada*, plusieurs conférenciers n'hésitaient pas à affirmer que $60 \%$ à $80 \%$ des motifs pour chosis une destination porteraient dorinarvant sur laspéaficiaté et la qualité del'environnement régional et local.

Dans ce contexte, il devient alors évident que déjà, et sans trop bien en saisir la portée et les implications, l'ensemble des régions et localités se situent dès maintenant en compétition environnementale à l'échelle québécoise et internationale et il est probable que cette fois, ce sera le touriste quí jugera des régions belles et moins belles.

Rien que ce fait devrait, au cours des prochaines années, inciter les régions à une sérieuse objectivité d'appréciation de leurs milieux respectifs et à une offre de services basée cette fois sur $l a$ diffórenciation plutôt que sur un concept d'égalite adninistrative. Il ne s'agira donc phus de s'exprimer à la place du dient touriste mais, de tenir compte de ce que ce dernier extimera beau, qualitatif, autbentique ou pas!

Ce revirement de situation entraînera probablement la nécessité d'une toute nouvelle géographie touristique, où la qualité des accès routiers à une région ou à une ville devront prendre la forme de *portes d'entrées environnementaless, où les circuits seront surtout panoramiques, et où les coquilles environnementales de séjour devront être des îlots qualitatifs culturels et naturels. Tout ceci entraînera les régions et les localités dans une course à $L a$ sélectivité et au réaménagement de certains sites plutôt que d'autres. La dispersion du produit touristique actuel fera danc place à la concentration et à l'efficacité de ce dernier dons certaines zones exceptionnelles.

Un autre effet qu'aura la volonté de vivre temporairement l'environnement biogéo physique et saisonnier sera probablement de redonner aux unités environnementales globalesunenouvelle réalité. Sans remettre tout à fait en question l'approche par activités des années 1990 , il semble qu'une forte tendances'effectuera vers un nouveau type de destinations environnementales. Compte tenu du fait que l'on pourra s'héberger et se restaurer, effectuer un circuit, faire de la motoneige, chasser et pêcher, faire du ski, tenir un congrès... à peu près partout au Québec, $k$ touriste choisira à lavenir un type d'environnement global phutôtt qu'un autre pour $y$ effectuer son expérience devie, Cettetendanceestdéjà trèsmarquée aux États-Unis, en Europe et mềme en Asie, peu importe la méthode employée, du *back packings, au séjour dans un *resorts luxueux.

Àl'aubedel'an 2000, la volonté touristique aboutiraainsi probablementà desintentions de fourisme maritime, de tourime foretier, de tourisme champêtre, de tourisme nordique (plutôt que d'aventure), de tourisme culturd urbain, etc. Cette «nouvelle s approche, qui rejointd'ailleurs étrangementcelledu XIX ${ }^{c}$ siècle sous une autre forme, interpelle le tourisme interrégional, en ce sens qu'il obligera rapidement à choisir, à se différencier et à se «spécialiser»d'une région à une autre de façon complémentaire et saisonnière.

Un autre aspect important touchant l'environnement concernera cette fois le réaménagement des lieux touristiques par *gisements environnementauxo ${ }^{(9)}$ ou concentration du produit à certains endroits précis danş les régions ou même au sein des localités. À cet effet, une des illusions des années 1970/1980 aura été de s'imaginer que le touriste est manipulable à volonté dans l'espace et qu'il peut être dirigé partout.

La réalité des années 2000 etplus sera toute autre! Il est bon de rappeler que le biocomportement humain répond à des nécessités objectives qui font que le touriste, ayant quitté son territoire habituel, cherche ailleurs des sites à haute teneur attractive. Il se dirige alors naturellement vers des lieux-cibles contraints où il est assuré de découvrir un maximum concentré de Culture/Nature. 
Ce phénomène, qui répondra du même coup à la nécessité de rentabiliser au maximum sestemps devacances de plus en plus courts, ira en augmentant et amènera les $M R C$ et les localités, non seulement à des choix stricts de lieux touristiques catalyseurs mais aussi, à des réaménagements majeurs par déménagement et rassemblement des attraits.

Dans un contexte futuriste, anticiper toutes les conséquences qu'il aura à agir de fapon concentréeplutôt que dispertée commec'est actuellement très souvent le cas, est hasardeux. Malgré tout, on peut déjà avancer l'idée qu'il y aura nécessité pour plusieurs territoires, villes et villages de s'unir pour aér des points d'attraits forts et significatifs dans l'espace commun. En d'autres termes, il faudra inverser le raisonnement actuel et cesser d'imaginer que tout est beau et économiquement rentable partout et n'importe où, et que le touriste est prêt à se déplacer comme client, à la porte de tous les visités.

\section{Les nouveaux environnements... sites du futur, et produits régionaux uniques}

Ce qui précède pourrait laisser croire, qu'à part certaines régions et localités déjà privilégiées, il n'existequ'un *no future pour celles qui héritent des *ravages» industriels etdes laissés-aller du XX' ${ }^{*}$ siè̃cle. Rien n'est plus faux, mais à ce chapitre, le Québec accuse un retard important et aurait une tendance un peu trop rapide à agir par exclusion comme le précise Bernard Vachon (1993) de l'UQAM. Álinverse des idées reçues, les environnements les plus performants du XXI' siècle pourraient êtreceux qui aujourd'bui apparaissent détériorés, négatifs et abandonnés. Ceux qui nécessitent le plus d'innovation réelle pour basculer dans lan 2000 plus. Car, à vrai dire, il n'y a rien de bien neuf à vouloir ramener un site à son état «naturel». La rélle innowation est celle qui consistera à faire passer un champ, une carrière, une gravière, une usine, une mine à ciel ouvert, wn village entier, etc., dans le futur en lui ajoutant une valeur et une bonne dase de culture, d'art et de tecbnologie avancée ${ }^{(10)}$.

Àcet effet, nosrecherches nousont amenés à découvtir quedans denombreuses régions américaines et canadiennes émergent de nouveaux types d'environnements qui suscitent le plus grand intérêt chez les touristes. Qu'ill s'agisse des monumentales gale-

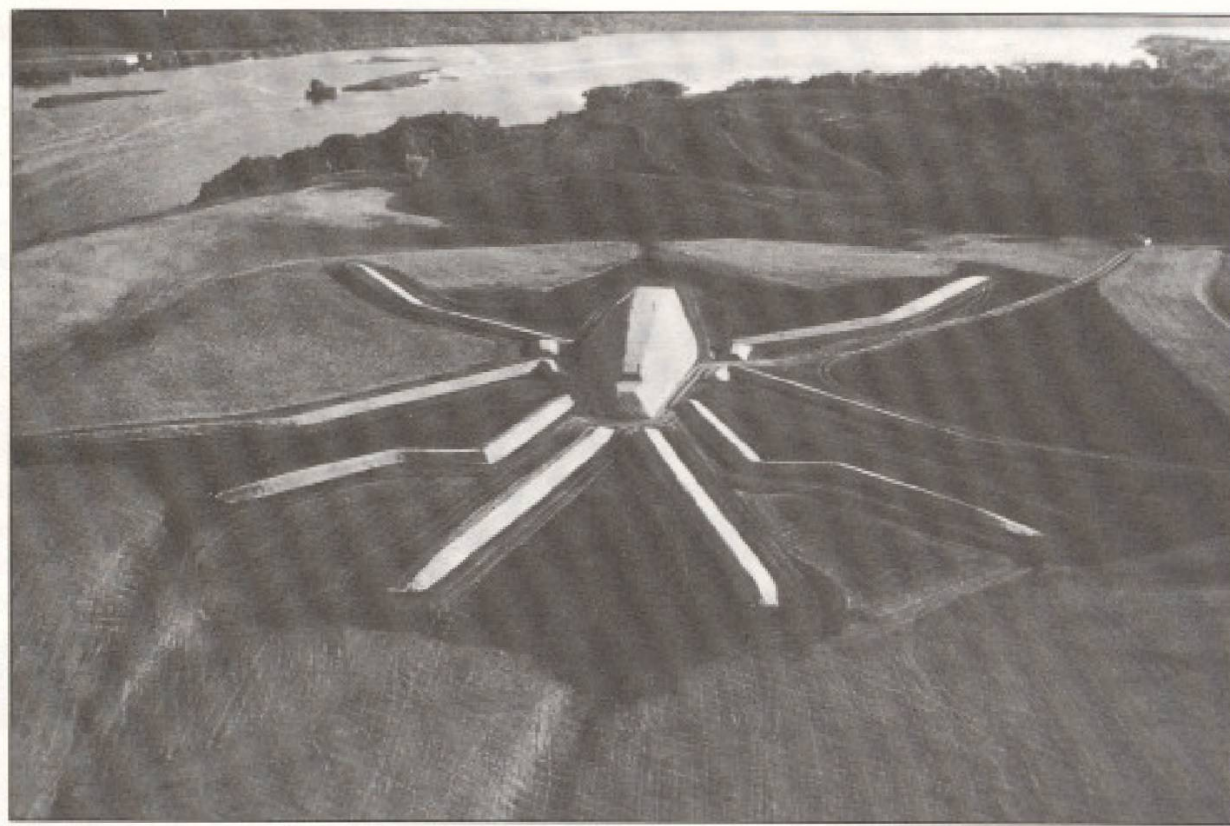

Le pues drau. Insecte symboliquo, devenu une colossab sculpture en forme de tumul. A rorigine mine poluante d ciel ouvert, le site a btb entibrement roconstitud a peu de frais pout devenir un parc boologiquo, macro-artistique et culturel, une sorte de monument pour le 3ieme millenaire qui mesure $695 \times 90 \times 14$ pieds. Phote : Michael Heizer, Water Strider of the Effigy Tumuli Sculpturea, llinois 1965.

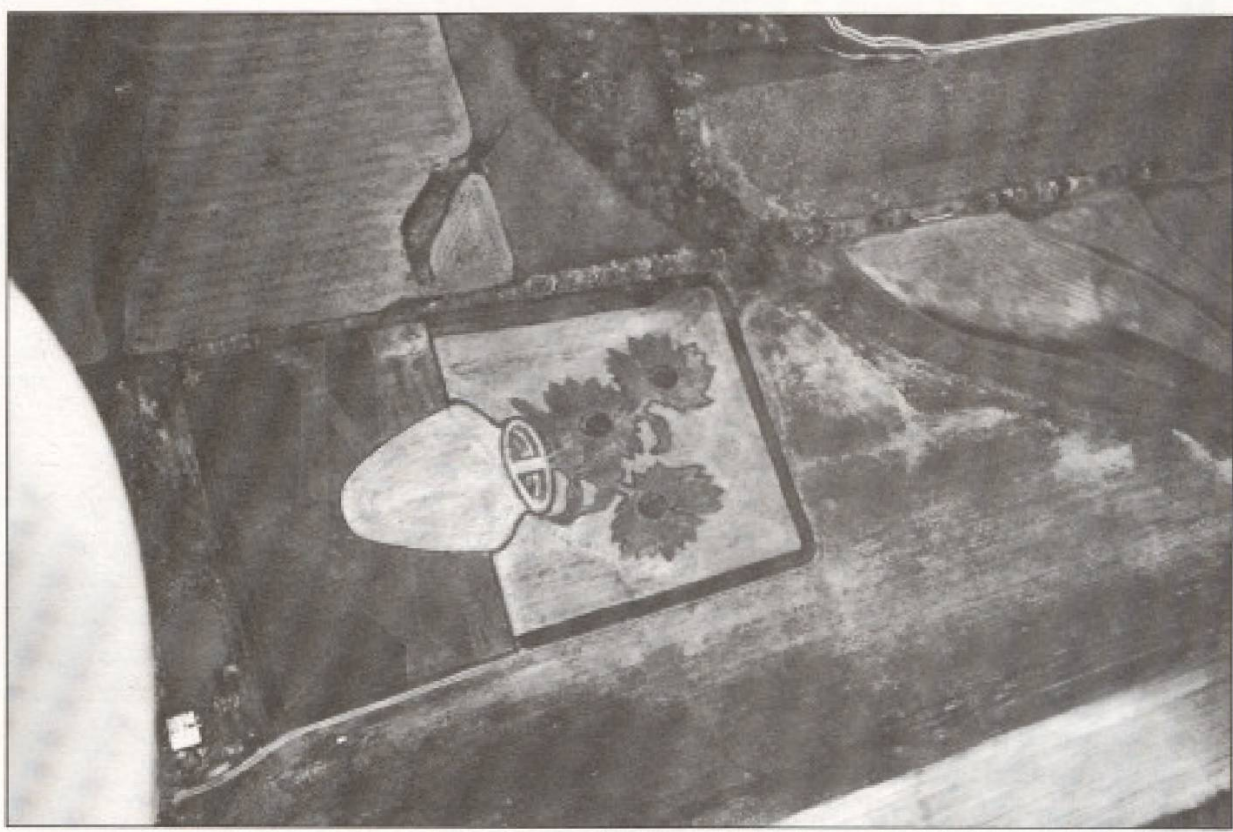

L'mgriculture de paysage ou ... comment cultiver asthetiquement les champs de l'an 2000 plus. En sgrotauriame, il ne s'agit plys simplement d'entretenir l'image bucolique du passe mais de taire basculer des espaces agraires abandonnes dans le 3ieme milienaire on innovant et en crbant de nouveles attractions spectaculaires. Pheto : Jon Blume, Kansas, 19e5, Van-Gogh Sun Flowers by Stanley Herd.

ries d'art de Chemainus en Colombie Britannique, d'Athens et de Welland en Ontario, ou des champs dessinés et ensemencés par Stanley Herd au Kansas, ou encore, des tumuli de Buffalo Rock en Illinois, tous indiquent une voie prometteuse pour les quinze à vingt prochaines années. En fait, ils représentent le pendant des paro naturels que l'on continue à multiplier de facon plus ou moins utile et ils sont en quelque sorte des para du futur que l'on devrait mettre, ici même, en place dans certaines régions ${ }^{(11)}$.

\section{L'écotourisme... phénomène à plusieurs volets}

Branche particulière du tourisme des années 1980-1990, ce domaine est en fait très ancien et remonte à l'antiquité. Il est probable que son intérêt se poursuivra au- 
delà de l'an 2000, sans pour autant devenir... Le tourisme du futur. Là comme ailleurs, les régions et les zones qui posséderont des écosystèmes rares, spectaculaires et très perceptuels pour le touriste, incluant les façons de vivre particulières de petits groupeshumainsdansleur milieu, pourront au cours des dix ou quinze ans à venir anticiper un développement viable. En fait, c'est l'écologie bumaine de type socioculturel, associée au paysage, au végétal et à l'animal qui dominera.

En conséquence, il sera intéressant que la plupart desparcs situés en régions prévoient la réinsertion partielle du *geste humain» permanent au sein de l'environnement végétal et animal disponible. Ce quidevrait corriger dans un avenir prévisible, la *déportations et l'expropriation effectuées au nom de la «nature pures et qui ont eu lieu au cours des années 1970-1980. Quant au potentiel de rentabilité de l'écotourisme, celui-ci sera toujours limité, comme l'indique certaines recherches effectuées par l'Université McGill(12)'

Il faudra aussi prévoir qu'au cours des prochaines années, un noyau dur de l'écologisme radical concernant la défense des animaux, celle des mammiferes en particulier, soit très agissante. Après avoir atteint ses objectifset fait interdire la chasse aux phoques pour la transformer en observation, la «deep ecology» abordera avec efficacité une action anti-chasse. Les résultats de cette stratégie seront importants pour les régions périphériques et les centaines de pourvoiries concernées. Car, la chasse sera graduellement associée à un acte de violence meurtrier, gratuit et contestable, au sein d'une société de plus en plus urbanisée. En retour, les nombreuses associations de chasseurs devront connaître et se former aux mécanismes que la *deep ecology» utilisera pour les attein$\mathrm{dre}^{(13)}$. Dans ce domaine, le «Future Pulls exigera une mutation des actions actuelles et une ouverture sur un nouveau tourisme forestier plus observateur que prédateur.

\section{Produit touristique... du déclin de I'un à l'émergence de l'autre}

La prise de conscience environnementale mondiale des dernières années a entraîné, surtout en Occident, des comportements sociaux informels qui influenceront dorénavant directement les produits touristiques régionaux et locaux. Sans vraiment le formuler, nous sommes collectivement en train de vouer au déclin des produits du $\mathrm{XIX}^{\mathrm{c}}$ et $\mathrm{XX} \mathrm{X}^{\mathrm{e}}$ siècle et d'en faire émerger de nouveaux plus complexes et plus respectueux de la vie en liberté, plus perceptuels aussi! Le toutaidé par la haute technologie des communications.

Un des exemples les plus frappant de cette mutation concerne les zoos : tout d'abord appuyé sur la mythologie grecque et les combats des Hommes-Dieux contre des animaux fantastiques, puis, prolongés par les exploits des rois qui capturaient lions et tigres en vue de démontrer leurs forces sur la nature, les zoos sont maintenant perçus comme des lieux contraignants au sens figuré et propre du terme $e^{(16)}$. On leur préfêre un concept avancé, celui du *World life Center». II s'agit donc d'un produit en déclin qu'il faut, soit abandonner dans sa forme passée, soit transformer, pour le futur.

Le zoo de l'avenir sera sans barreaux ni animaux, de prévoir le concepteur du nouveau zoo de Londres fermé après 165 ansd'opération. L'alimentation des animaux à trois beures de l'après-midi sera remplacée par une liasison satellite avec la forêt tropicale de lAmazonie, la Grande Barrière de récifs de lAustralie, l'Antarctique ou l'Afrique, de facon à permettre aux visiteurs d'interagir avec differents environnements... Mieux encore, une des zones principales du nouveau zoo consistera en un «sensorium» où on recréera la vie telle que perçue par les animaux...

À l'inverse des produits régionaux qui déclinent et dont on doit reconnaître un jour qu'ils sont dépassés par l'évolution et les attentes des clients, d'autres émergent et connaissent des succès fulgurants dans des domaines culturels inattendus. Tel est le cas par exemple de la monumentale fresque théâtrale populaire et locale, intitulée \& $\mathrm{La}$ fabuleuse histoire d'un royaumes au Saguenay, ou celui desmusées vivants dont le Québec est malheureusement encore dépourvu.

Ce qui précède indique brièvement, mais de façon claire, que beaucoup de régions seront soumises à un effet de cisaillement entre les produits en dédin et ceux qui émergent. Le choix sera souvent douloureux car, il exigera des localités de prendre connaissance du futur avant d'agir et de s'inscrire résolument au sein de la mondialisation et de l'internationalisation des marchés... pour réussir.

En un sens, le moindre produit touristique local devra dorénavant prendre conscience du pouls du monde et se soumeture à la concurrence des idées. II devra aussi être à la fois endogène quant au pouvoir de se développer et exogène pour certaines des connaissances à appliquer ${ }^{(15)}$. Dans ce contexte, les régions qui choisiront d'être l'un et l'autre risqueront souvent de perdre beaucoup de temps à tout réinventer et de ce fait, elles seront peu concurrentielles et performantes sur l'ensemble des marchés nationaux et internationaux. De plus, elles engloutiront des capitaux... sans résultat!

Enfin, un autre phénomène concernera les réseaux de services d'hôtellerie, de restaurationetd'activités. De plus, ceux-ci seront obligés de s'intégrer dans un système de communication global permettant au client de choisir les qualités environnementales de son séjour à desmilliers de kilomètres de distance. En laan 2000, plus on lowera systématiquement une chambre ou une table de restaurant en fonction de son orientation at plus on choisira une localité selon la perception que l'on a d'une activité et d'une expérience à y vivre, ceux qui ne pourront y répondre seront déclassés et exchus du marcbé.

\section{Choisir le bon client touristique... "baby boomer et empty nest"}

L'industrie touristique des années 20002005, contrairement au loisir touristique plus ou moins subventionné des années 1970-1980, est déjà à la recherche d'une clientèlestable etéconomiquementnantie, qui permettra une rentabilité directe des environnements, des infrastructures et des services.

Ceci ne veut pas dire pour autant qu'elle sera exploitable à outrance! Bien au contraire, le rapport qualité/prix aura une grande importance pour ces personnes ce qui devrait, dans bien des cas, modérer un certain *cannibalisme du portefeuille touristique dans certaines régions et localités, du moins si on souhaite que cette clientèle soit durable et renouvelable.

Comme le précise Simon Langlois dans une Ebarucbe des tendances lourdes du système québécous - une société et une culture sous tension (1993), il y a en ce moment un déclassement d'une fraction de la classe moyenne. 
La période du nid vide (empty nest) s'est allongée, de même que celle de la retraite active et il $y$ a ếmergence d'un quatrième âge. La classe moyenne des $28 / 40$ ans s'est affaissée au centre.

Malgré tout, cette mutation est loin d'être financièrement négative dans le cadre futur du tourisme et les prévisions générales à la hausse de cette industrie n'en seront que peu affectées. Il s'agira dorénavant de viser les bonnes clientèles et surtout d'adapter la majorité des produits actuels à cescatégories d'àge. Là, à notreavis, réside la principale mutation qui exigera dela part des régions beaucoup d'innovation pour s'adapter au troisième millénaire. Car, nombre d'entre elles sont encore malheureusement axées sur la lancée des années $1980^{(16)}$, ou encore, croient s'adapter au futur en traitant du produit touristique pour... un *troisième âges physiquement ou intellectuellement amoindri. Ce qui est pire que tout comme approche!

Simon Langlois spécifie encore: «... en $1971,10,7 \%$ de la population vivait en couple sans enfant présent à la maison; en 1991, la proportion atteint près de $20 \%$. La très grande majorité de ces couples vivent seuls... Il est entendu que plusieurs couples peuvent avoir des enfants qui ont quitté la maison, surtout après 55 ans... Le revenu réel disponible a baissé en 1990 , comparé à 1978 d'environ $10 \%$ chez les moins de 40 ans alors qu'il augmentait dans les familles dont la personne de référence avait plus de 50 ans. C'est donc dans cette stratede clientèle que le tourisme puisera à l'avenir ses ressources financières.

En plus des nouvelles clientèles à bien servir, il faudra prévoir que les trois régions frontalières avec les États-Unis: l'Estrie, la Montérégie et Chaudière-Appalaches, tisseront des liens privilégiés avec les états contigus de façon à créer un marché et une unité territoriale particulière.

Enfin, pour les villes comme Montréal, Québec, Trois-Rivières... il est probable que le tourisme dirigé et organisé par les minorités ethniques asiatiques, américaines et autres, en relation directe avec leurs pays d'origine, pourrait connaître un essor sans précédent.

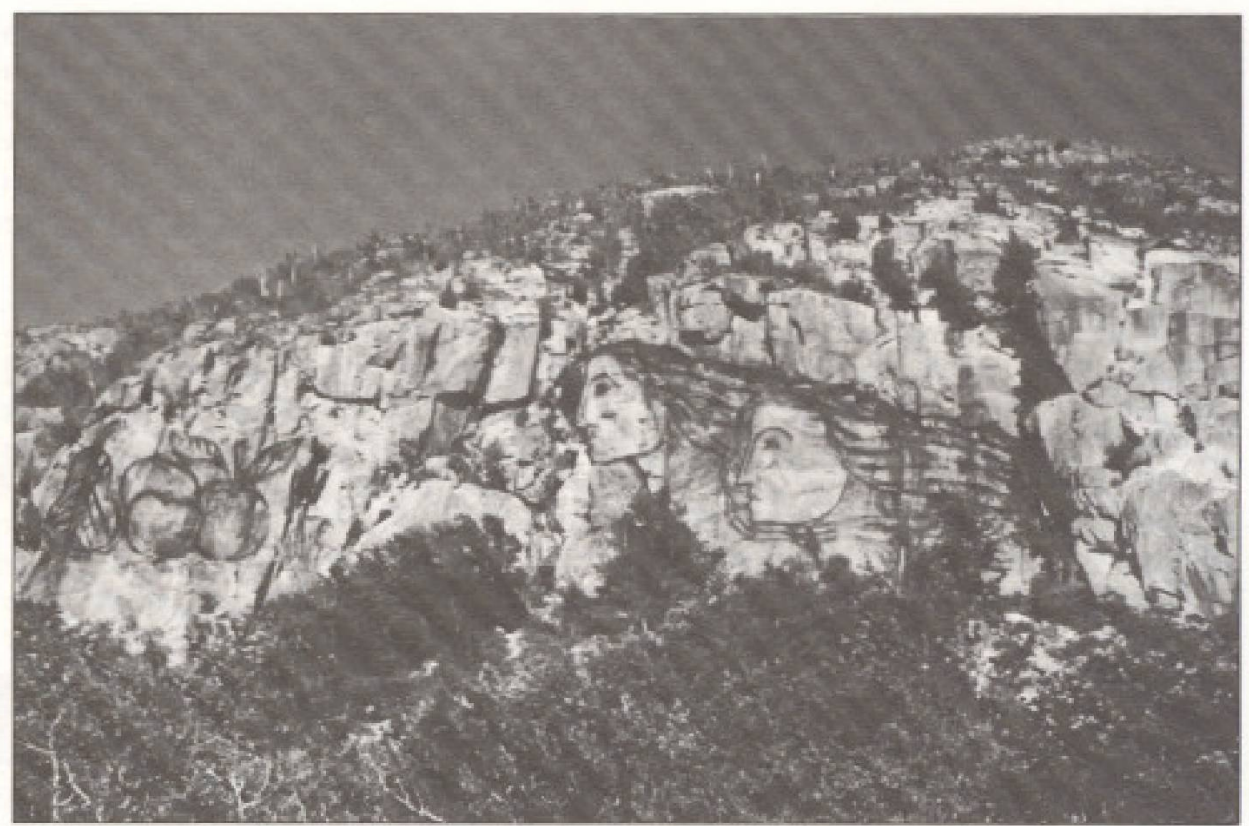

Le Cosple. Element central Culture - Nature. Cette gigantesque peinture rupestre, presque transparente at diacrbite, faìt partie du projet touristique global de laville du Mont St-Hilaire. Conçue par les mèmes auteurs que cet article, les profils soulignent les fomes de lareche et prolongent lhistcine du lieu qui a vu naltre PaulEmile Berduas et une partie du arefus globals. Phote : Eugen KEDL. Oubbec. 1990.

\section{Le positionnement culture- nature... ni parc, ni musée}

L'intéressant positionnement culture-nature du Québec, inscrit dans la récente politique touristique, devrait déjà amener les villes pivots et certains espaces régionaux naturels, forestiers et agricoles à envisager le futur sous des angles complémentaires à la métropole, Montréal, et à la capitale, Québec. Des angles, où La reconduction systématique du modele culturel urbain par excellence: $l$ musée, et la représentation de La nature dans l'espace régional: le parc, seraient choses du passe ${ }^{(17)}$.

Ce scénario prospectif est déjà en émergence, par exemple à North Hatley, qui a choisi avec beaucoup de justesse d'être le lieu global de l'art - d'artisanat naïf international - ou à Joliette, avec le festival de musique classique en plein air - ou encore à Mont-Joli avec la galerie d'art environnementale du peintre sculpteur Gagnon... Toutceciayant pour objectif de créer des attractions uniques qui donnent déjà à certaines régions et localités une originalité concurrentielle propre aux caractéristiquesdel'espace régional, quin'est pas celui de la ville, faut-il le rappeler!

Associés à la construction des enouveaux environnementsw, nous avançons alors l'idée que le produit touristique régional et local deviendra hybride et personnalisé et que de ce fait, il correspondra mieux aux types de clientèles des 40 à 65 ans. Une catégorie d'individus qui, en pleine maturité physique et intellectuelle, ne souhaiteront pas se rendre en régions pour $n^{\prime} y$ voir qu'une grande nature sans culture ou une pâle culture calquée sur la grande ville.

En conséquence, les régions se retrouveront devant un avenir prometteur, pour peu qu'elles sauront se créer un univers propre et situeront leurs actions dans un cadre inusité. D'ailleurs à l'inverse, c'estce que Montréal a déjà réalisé en érigeant son Biodôme... Nature-Culture. D'autres dérivés directs de l'approche intégrée culture-nature s'appliqueront en région dans des domaines aussi précis que l'hébergement, la restauration, les activités et les échanges sociaux.

Le touriste des années 2000-2010 voudra de plus en plus habiter la culture (la vivre) en dormant dans des édifices patrimoniaux de type auberge de caractère comme le préfigure si bien le réseau qualitatif des *maisons d'antan\%. Quant à l'hôtellerie régionale des années 1980-1993, celle qui ne possède que peu d'originalité architecturale et déjà une mauvaise situation environnementale dans le paysage, elle aura beaucoup de difficulté à survivie; le tourisme d'affaire diminuant sans cesse au profit des télé-conférences et de la rationalisation des entreprises et les nouvelles clientèles n'acceptant plus de dépen- 


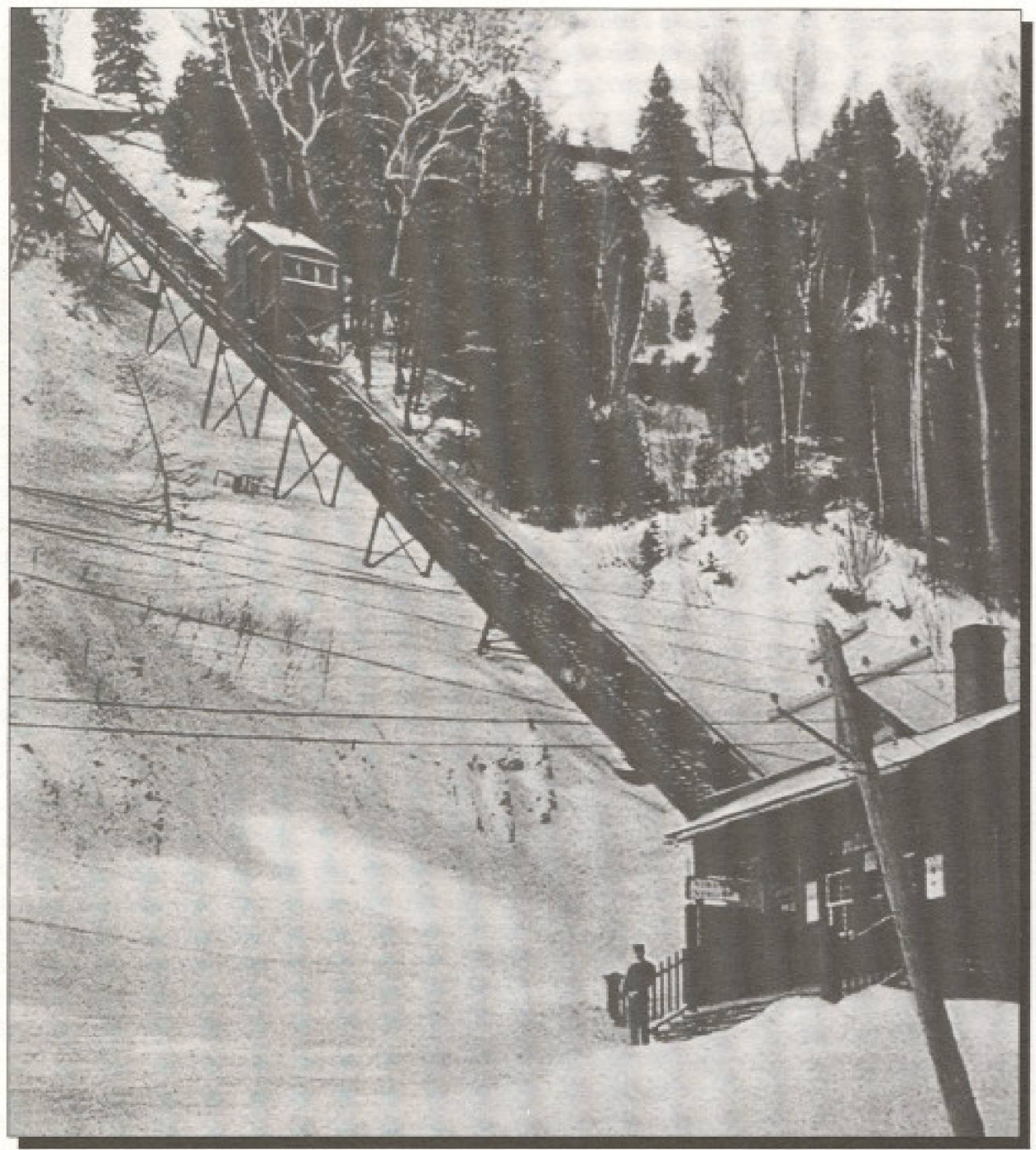

Le Pare de le Chute Montmoraney $\$$ Oubbe. Enfin le futur, devrait-on dire, en considerant la valeur ajoutbe locomotrice a la nature du site I Mais malheureusement, une intbgration du tolepherique a l'environnement qui laisas beauseup a dbsirer wec des fils qua decoupent le panorama, un batiment qui l'obstrue et une tachnologie en rupture historiaue.

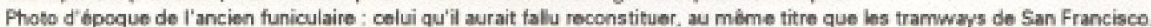

ser $9 / 10 \mathrm{e}$ de leur budget journalier pour juste dormir et se nourrir le soir.

En fait, c'est le Québec profond de la qualité qu'il s'agira de remettre en valeur partout où il n'existe pas déjà. Quant à la relation sociale que le touriste voudra entretenir avec les habitants des régions, celle-ci portera surtout sur l'écologie humaine et donnera naissance à une sorte d'ethnotourisme. Mais plus encore, le touriste exigera au cours des quinze prochaines années que la technologie des communications, associée à la culture du $\mathrm{XXI}^{e}$ siècle, le réintègre dans les espaces «naturelss ambiants des régions et lui procure une expérience de vie passée, présente et future à la fois. Et cette expérience de vie se situera à des niveanx perceptuels et sensoriels plutôt qu'intellectuels et plysiques.

\section{La locomotion... lien environnemental indispensable de I'espace touristique}

Au cours des années à venir, la locomotion intếgrée et polyvalente devra occuper une place tout à fait privilégiée et prépondérante dans l'industrie touristique pour parcourir, traverser, pénétrer, survoler, mais surtout interrelier les environnements terrestres, aquatiques et aćriens de lespace touristique. Nous pourrions même dire qu'il est probable que le phénomène locomoteur dans son ensemble deviendra aussi im= portant que l'a été celui des infrastructures lourdesau cours des trentedernières années. Cela concerne à la fois les voies de lacomotion, mais atussi les moyents de locomotion, en partant du trottair et du sentier de marcbe, de la piste cydable courte et de la bicydette, jusqu'aux bélicoptères, funiculaires, tramways, bydravions, paquebots géants, sous-marins, montgalfières, etc. ${ }^{\text {(a) }}$

En fait, le réseau locomoteur et la diversité locomotrice d'une localité ou d'une MRC par exemple, devra procurer à l'espace touristique futur une cohésion générale, en lui octroyant une valeur ajoutée quasi instantanée et des possibilités d'entreprises immédiates. Actuellement, dans ce domaine, seules les villes de Montréal et Québec ont vraiment abordé le phếnomène avec les hauts et bas engendrés par un développement peu planifié etmal compris. La*crise denerfsćcologiste» dela région de Québec, qui découvre subitement les bienfaits économiques et les inconvénients *normaux du transport aérien touristique, en est un exemple marquant!

Il faut le reconnaîtresans détour, leQuébec, dans le secteur de la locomotion touristique et des moyens de transport propres au mouvement de la personne dans l'espace, devra agir en rattrapage pendant au moins une bonne décennie. Car, cet item devra être considéré comme indispensable à la cohésion globale des produits touristiques régionaux, et il ne devra plus être confondu avec une activité secondaire.

En ce sens, l'exemple des tramways de San Francisco est significatif et devrait encourager les capitales régionalesà sedoter d'un minimum de transport collectif touristique. Car, le touriste souhaite être pris en chargeà la sortiede sa voitureetdel'autobus, et il accepte facilement d'être guidé et transporté à travers l'espace touristique 
culturel et naturel. Rien que l'application de ce principe, s'il est bien structuré, peut dans certains cas, aider substantiellement le commerce de détail. Rappelons qu'à San Francisco, la flotte de tramways et funiculaires qui transporte presque uniquement des touristes à 3,00 \$ le passage, rapporte environ 10,5 millions, pour 16 kilomètres de parcours. Elle est aussi une des principales attractions majeures de la région, elle-même basée sur le patrimoine technologique.

Bref, si la locomotion touristique doit dans un futur proche combler un manque et relancer certaines agglomérations et sousrégions, il n'en reste pas moins qu'elle devra aussi se concrétiser au travers de moyens et principes de locomotion appropriés et soigneusement intégrés. En ce sens, il ne serait pas surprenant qu'au cours des prochaines dix années, les régions se dotentd'un authentique réseau locomoteur intégré, instrument indispensable à toute industrie, au même titre que celui des communications et des réservations pour le tourisme.

\section{Formation en tourisme... transférer la bonne connaissance, à la bonne personne, au bon endroit}

Si l'on souhaite au cours des deux prochaines décennies, que l'industrie touristique soitplusnovatriceet doncplus performante, génératrice d'emplois directs et d'entreprises rentables dans les secteurs primaires et secondaires et tertiaires ${ }^{(20)}$, il s'agira à brève échéance de réviser à nouveau la formation. Car, à l'exception de Montréal et de Québec, de certaines villes pivots et localités qui possèdent déjà une masse critique d'entreprises touristiques florissantes et qui nécessitent un perfectionnement, les besoins en formation technique (accueil, etc.) sont dans la réalité, restreints. Ils poutraient être assumós à même l'entreprise et n'exigent pas qu'on Lur consacre antant de ressources bumaines at financières et encare moins une structure éducative permanente au sein d'une maison d'éducation.

En réalité, ce ne sera pas le jeune cégepien qu'il faudra surtout former à l'avenir(al) pour qu'ensuite il ne trouve que difficilement un emploi dans une industrie touristique régionale de plus en plus faible, mais des adultes responsables, au sein des localités en déstructuration. Des gens, dont lemploi est devenu précaire, qui possièdent une maison, une crédibilité dans leur village ou quartier, et qui ont un besoin urgent de connaiosances directes pour diversifier immédiatement leur revenu et rester en place. En un sens, là comme ailleurs, il est probableque la formation en tourisme s'éloignera d'une certaine «bureaucratie éducative, coûteuse et lourdes, et qu'elle tendraà s'adresser directementà la personne danssa localitéetson milieud'appartenance réel.

Dans ce contexte, il est fort probable que deux grands types de formation seront nécessaires au redéveloppernent touristique régional et local. L'un à caractère populaire et général qui s'adressera à un maximum de décideurs régionaux pour leur faire connaître ce qu'est l'industrie touristique dans son ensemble, avec ses intersectorialités. L'autre, plus spécifique, quis'adressera à l'entrepreneurship local et qui visera à l'accompagner dans le montage d'entreprises touristiquesspécialisées. L'un pourra être assuré par une éducation à distance comme la télé-université etl'autre, par les CADC et les $\mathrm{CFP}^{(2)}$.

En somme, ce qu'il s'agira de choisir au cours des années 2000 plus, ce sera de continuer à former une main-d'oeuvre dépendante d'une industrie à rénover, ou encore, d'octroyer des connaissances adéquates à des entrepreneurs locaux pour qu'ils puissent amorcer la relance du tourisme et engendrer des emplois qualifiés dansles localités etrégions d'appartenance.

\section{Conclusion}

Alors que les scénarios prospectifs des années 1980 étaient surtout catastrophiques et apocalyptiques, ceux de 1993 ressemblent plus à des hypothèses permettant d'établir des fourchettes d'options. On les appelle des scénarios tendanciels et exploratoires et tous font référence à une ou plusieurs problématiques sectorielles qu'ils'agira de corriger dans le futur de 2000 plus.

En principe, l'industrie touristique des années 2000/2010 devrait être au Canada et au Québec synonyme de croivance économique, de creation d'emplois directs et d'entreprises hucratrives. Nous disons en principe, car malheureusement il se pourrait que ce ne soit pas le cas. Alors que la courbe du tourisme mondial est ascendante, la nôtre chute. Ceci est anormal pour le Canada, pour le
Québec, pour les régions et les localités. En somme, notre perception de l'avenir touristique se trouve une fois de plus basée sur le paradigme du $*$ rattrapage $»$, soitcelui quiaguidéla plupart des actions du Québec depuis 1960.

Toutefois, en analysant la situation touristique industrielle présente pour en dégager des axes futorogènes, nous avons refusé la démarcheconservatrice, quiauraitconfirmé du même coup notre recul dans un domaine prometteur..., ailleurs. Une démarche endossée par certains prospectivistes qui annoncent que, sans trop risquer de se tromper, on peut prévoir que le Québec de 2010 ressemblera considérablement au Québec de 1993, lequel ressemble encore à celui d'il $\mathrm{y}$ a treize ans, soit 1979 !

Une telle approche fataliste ne nous convenant pas, nous avons envisagé le futur touristique des régions du Québec comme pouvant résoudre les oontradictions actuelles et comme étant capable de concurrencer d'autres régions sur le marché international. Notre prospective optimiste et dynamique en est donc une à double action. Toutefois, dans la mesure où cet effort de tous et chacun n'aurait pas lieu pour des raisons diverses, ce sera alors à notre tour d'être graduellement exclus du tourisme futur et de son important apport économique régional local.

En conséquence, les options pour l'avenir en matière d'industrie touristique sont une sorte de non choix à double effet. Ou bien le tourisme se resitue au centre et non à la périphérie de l'exploitation sélective de sa matière première que sont les environnements culturels et naturels et de l'esthétique générale; ou, il continue de se placer trop exclusivement dans le secteur tertiaire des services. Dans ce cas, il ne pourra faire face à la concurrence de l'Europe, des Etats-Unis, ni même de l'Asie qui, eux, utilisent directement des sites patrimoniaux etnaturels à haute valeur ajoutée. Une fois cela accompli, ou le tourisme canadien et québécois feront en sorte d'exploiter l'environnement en lui ajoutant une valeur commerciale et manufacturière en transformant en objets divers les caractéristiques du milieu; ou alors, les milliers d'objets souvenirs reliés à l'art-d'artisanat continueront d'être inexistants ou importés, créant des emplois à l'extérieur.

De plus, ou le secteur des services, celui de l'hébergement, de la restauration et des 
activités, acceptera d'abaisser ses prix et de jotuer la carte de la qualité totale en misant sur un afflux supérieur de clientèles nanties; ou, le tourisme interrégional et international ira en s'amenuisant au Canada et au Québec ne pouvant faire face à la concurrence. Enfin, ou la formation en tourisme, dé de voüte du développement local, s'adressera directement aux localités en déstructuration et aux entrepreneurs adultes des régions; ou, l'éducation en tourisme continuera dans la majorité des régions, à enseigner des techniques plus ou moins appropriées à une industrie en déclin. $f$

\section{Notes}

(1) A considérer comme un fait et non une critique.

(2) A ne pas confondre avec les villages historiques reconstitues ou les aliving museum* americains de type Mystic Seaport dans le Connecticut aux Etats-Unis.

(3) Entendre par Sud, le Sud hivemal, comme la Floride l'hiver, le Sud printanier, comme New York at le Sud estival, comme la Nouvelle-Angleterre.

(4) Agir par exclusion de ce qui n"est pas qualitatif et concurrentiel et de ce qui se rapporte au loisir muricipal et non au tourisme rentable.

(5) Transformation de l'environnement culturel et naturel en biens et services * souvenirs: pour accroitre le secteur secondaire manulacturier, relie au tourisme.

(6) Pour l'avenir, I'idé de masse tendra d se fractionner en 6ibment amassisa plus restreints: les agregats, tournes wers la croisidre, l'aventure douce, etc.

(7) L'activité sera remplacoe par l'expérience de vie.

(19) Matiere premiere du tourisme des annees 2000 plus.

(9) Points de concentration, faunique, floristique, architectural dans les espaces régionaux.

[10] Le futur environnemental ne peut être envisagé quecomme une progres sion culturelle et corrective et non comme une régression vers un simple état de nature.

(11) Actuellement, qui dit environnement emploie le concept du *back to the future plutôt que celuidu sFuture pulla

(12) En relation avec les capacites de charge des écosystèmes visités.

(13) Ces organisations devraient trtre informees des strategies utilises contre elles par la *deep ecologyw et savoir cornment pro-agir. L'information devrait être faite sur une base d'éthologie humaine.

(14) II en est de même des aquariums classiques, des centres d'interprétation, etc.
(15) Le développement uniquement endogàne est un leurre aussi important que l'a été celui purement excgene qui l'a précédé.

(16) Les années 1970-1980 étaient basées sur le conditionnement physique, le sport et l'activité physique des moins de 40 ans.

(17) Les régions devront constituer un produit unique, non dichotomique.

(18) De plus en plus la technologie permettra de concrétiser les mythes relés a la nature of a la culture et de les offrir aux touristes sous forme spectaculaire tout en ameliorant les environnements.

(19) La bocomotion est le lien obligatoire qui donne un sens opérationnel aux infrastructures.

(20) L'industrie touristique devra a l'avenir s'appuyer a la fois sur la ressource primaire (paysage, site, etc) sur le secondaire manufacturier et le tertiaire des services pour se relancer.

(21) Quelques cours, dans des formations deja existantes devraient sutfire.

(22) Comitéd'aide au dévelopeement des collectivités - CADC - Centre de formation professionnelle CFP.

\section{Bibliographie restreinte}

CONFERENCE BOARD OF CANADA, Exdusit, "Numéros successifts sur les tendances du tourisme international, canadien et dans les provincess, 21 numeros, 1992-1993, $62 \mathrm{p}$.

CONSEILS RÉGIONAUX DE DÉVELOPPEMENT DE L'ABITIBI-TEMISCAMINGUE ET DE L'Estrie, Plans strateglques de dibveloppement, 1993 . $149 \mathrm{p}$. et $191 \mathrm{p}$.

GOUVEANEMENT DU QUEBEC, Conseil executif, Secrétariat aux affares régionales - SAR, Dévelop. per les riggions du Qubbec, $1992,97 \mathrm{p}$.

JACQUES, Jocelyn, "Les rógions de l'an 2000 au Oubteck, Alocution de Baie-Comeau, Secrétariat aux affaires régionales, SAR, $1993,18 \mathrm{p}$.

JAY.RAYON, Jean-Claude, LEFEBVRE, Plerre et Michel THERAIEN, Lee róglons de ran 2000 , document exploratoire, Secretariat aux affaires régionales, SAR, Pecherche et Prospective, 1993, 23 p.

JAY-PAYON, Jean-Claude, Le tourlame, mise on at tuation ot problitmatiques intervectorielles ot Interréglonales, Secrétariat aux affaires régionales, SAR, Recherche ef Prospective, 1993, $18 \mathrm{p}$.

JAY-RAYON, Jean-Claude, en collaboration avec Brigitte Morneau, Objectif science, 6le congres de IACFAS, document d'appoint aux interventions, L. développement - aménagement récrétour istique riglonal ef local... ou Yassociation culture-nature dane un cadre diffeonomila-Éeologie, recueil d'articles, 1993, 91 p.

UNIVERSITÉ DU QUEEBEC A CHICOUTIMI, sous la direction de Jean-Luis Klein et Bernard Maltais, Lo rber botour iame, une nouvelle vole de déveleppement, Association professionnelle des géographes du Qubbec, 1989, 147 p.
LATOUCHE, Daniel et collaborateurs, Projet Oudbec 2000 * une prospective du Oubbec a raubs đun sutre sibele, document préliminaire, 1993, $382 \mathrm{p}$.

MINISTERE DU TOURISME DU QUÉBEC, Des askons t dee gens, bnonct de politique ot plan draction en matibre de tourleme, 1992, $96 \mathrm{p}$.

SAUVE, Robatt, Futur Pull, Rural Exchange, ANationalInternational Workshop, Hull, Ouebec, 1993, 30 p.

VACHON, Bernard, avec la collaboration de Francine COALLIER, Le d6veloppement local, theorle of pratiqus. Reintrodulre Thumein dane la logtque au developpement, Editions Gabtan Morin, $1993,330 \mathrm{p}$ 